\title{
Comparative Study of Silicon Detectors
}

\author{
C.P. Allier, H. Valk, J. Huizenga, V.R. Bom, R.W. Hollander and C.W.E. van Eijk \\ Radiation Technology Group, Interfaculty Reactor Institute, Delft University of Technology \\ Mekelweg 15, 2629 JB Delft, The Netherlands
}

\begin{abstract}
We studied three different types of silicon sensors: PIN diodes, circular drift detectors, both made at the Delft University of Technology (DUT), and Hamamatsu S5345 avalanche photodiodes. Measurements have been carried out in the same optimized experimental setup, both at room temperature and at low temperatures. Comparison is made for direct X-ray detection and CsI(TI) scintillation light readout.
\end{abstract}

\section{INTRODUCTION}

The interest in elementary particle detection, X-ray spectroscopy and scintillation-light detection has resulted in the development of new silicon detectors: the silicon drift detector (SDD) $[1,2,3,4,5]$ and the avalanche photodiode (APD) $[1,6,7,8,9,10,11,12,13]$. The SDD has developed rapidly and replaces now PIN photodiodes in several applications. PIN photodiodes and SDDs can be produced from $\sim 500 \mu \mathrm{m}$ thick silicon wafers. If the active area doesn't exceed a few squared millimeters, a resolution of a few hundred eV FWHM can be obtained at room temperature with these detectors [4, 14]. However the SDD is relatively 'slow' and for time resolved measurements, the avalanche photodiode which is based on a high field region for amplification is more appropriate. Due to its features of internal gain, fast timing and high photon detection efficiency the APD is now developed in order to replace the PMT in several applications [11].

In the following we shall focus on the energy resolution and the detection efficiency obtainable with relatively large detectors. The SDD we studied is a $33 \mathrm{~mm}^{2}$ active area and $500 \mu \mathrm{m}$ thick circular detector. The PIN photodiode is a 81 $\mathrm{mm}^{2}$ active area and $500 \mu \mathrm{m}$ thick detector. The Hamamatsu S5345 APD is a $5 \mathrm{~mm}$ diameter $\left(\sim 20 \mathrm{~mm}^{2}\right)$ blue enhanced APD with an estimated conversion layer of $25 \mu \mathrm{m}$ thick [15]. The photodiode and the Hamamatsu S5345 APD were coupled to a low-noise charge amplifier, the SDD to a forward biased FET charge amplifier [16]. A specially designed $1 \mathrm{GHz}$ JFET with integrated feedback capacitor has been glued close to the readout electrode of the SDD to minimize the stray capacitance. The signals were processed by a spectroscopy amplifier ORTEC 672 and fed into an ORTEC AD114.

\section{NOISE AND ENERGY RESOLUTION CONSIDERATIONS}

The application of silicon detectors is restricted by the signal/noise ratio. Although the mean energy to create an electron-hole pair in silicon is relatively small $(3.6 \mathrm{eV}$ [17]), the signal is low because, except for the avalanche photodiode, there is no internal amplification in the detector. The noise comes from the input transistor of the preamplifier and the leakage current of the detector and the gate of the input FET [18]. The noise of the input transistor can be modelled as a voltage noise source in series with the input impedance. The leakage current gives rise to a current noise source at the input of the preamplifier, parallel to the input impedance. Both noise sources contain besides the 'white' component a $1 / f$ component. The charge pulse signal generated by a detector must be amplified by a charge sensitive preamplifier. However the output signal of the preamplifier is still low, so amplification and noise filtering is done with a spectroscopy amplifier. The rms noise referred to the input as a number of noise electrons $N_{e}$ is given by [19]:

$$
N_{e}=\frac{1}{q} \sqrt{\frac{a}{\tau} C_{T}^{2} A_{1}+\left(2 \pi a_{f} C_{T}^{2}+\frac{b_{f}}{2 \pi}\right) A_{2}+b \tau A_{3}}
$$

where $C_{T}$ is the total input capacitance formed by the detector capacitance, the input transistor capacitance, the feedback capacitance and the stray capacitance. $a$ is the spectral density of the thermal noise of the JFET, $a_{f}$ the spectral density of the JFET $1 / f$ noise, $b$ the spectral density of the white parallel contribution due to the shot noise of the JFET and the detector leakage current and $b_{f}$ the spectral density of the dielectric noise. $\tau$ is the shaping time constant of the filter and $A_{1}$ to $A_{3}$ are the filter shape constants. For the PIN photodiode and the SDD, the energy resolution (in relative FWHM) is related to the rms noise taking into account the statistical resolution by:

$$
\frac{\Delta E}{E}=2.35 \sqrt{\left(\frac{N_{e}}{N}\right)^{2}+\frac{F_{f}}{N}}
$$

where $N$ is the mean number of primary electrons and $F_{f}$ the Fano factor, approximately 0.11 for silicon [20]. For an avalanche photodiode, taking into account the internal gain $M$ and the statistical fluctuation of the gain reflected by the excess noise factor $F\left(F=M^{0.28}[15]\right)$, the energy resolution is given by [12]:

$$
\frac{\Delta E}{E}=2.35 \sqrt{\left(\frac{N_{e}}{N M}\right)^{2}+\frac{(F-1)}{N}+\frac{F_{f}}{N}}
$$

When a detector is coupled to a scintillator crystal, the energy resolution (in relative FWHM) is given by [21]:

$$
\begin{aligned}
\frac{\Delta E}{E} & =\sqrt{R_{d}^{2}+R_{s}^{2}} \\
R_{s}^{2} & =R_{i}^{2}+R_{p}^{2}
\end{aligned}
$$


Table 1

Characteristics of the detectors at room temperature.

\begin{tabular}{|l||l|l|l|l|l|}
\hline detector & $\begin{array}{l}\text { active area } \\
\left(\mathrm{cm}^{2}\right)\end{array}$ & $\begin{array}{l}\text { detector junction } \\
\text { capacitance }(\mathrm{pF})\end{array}$ & $\begin{array}{l}\text { leakage current } \\
\left(\mathrm{nA} / \mathrm{cm}^{2}\right)\end{array}$ & gain & $\begin{array}{l}\text { optimum bias } \\
\text { voltage }(\mathrm{V})\end{array}$ \\
\hline \hline PIN photodiode & 0.81 & 20 & 0.8 & $/$ & -50 \\
\hline SDD & 0.33 & $<0.1$ & 3 & $/$ & -230 \\
\hline APD S5345 & 0.20 & 90 & 24.5 & $11-13$ & -260 \\
\hline
\end{tabular}

where $R_{d}$ is the detector resolution described by the equations (2) and (3), $R_{s}$ the scintillator resolution, $R_{i}$ the intrinsic resolution reflecting the non-proportionality and the inhomogeneity of the scintillator and $R_{p}$ the transfer resolution.

\section{Measurement of BAsic Properties}

From equation (1) it follows that the dominant contributions to the electronic noise are the total capacitance parallel to the detector and the total leakage current. These and other parameters are summarized in table 1. The PIN photodiode exhibits a low leakage current but shows a high junction capacitance. The SDD has a very small capacitance in comparison to the two other detectors. This requires a low capacitance preamplifier for optimal results [16].

In the APD, the gain and the leakage current grow exponentially with the bias voltage. A compromise between the noise and the gain is found at a bias voltage of $-260 \mathrm{~V}$. For calibration of the APD gain, the signals were compared with pulses fed through the test capacitor of the preamplifier. Measurements using ${ }^{55} \mathrm{Fe},{ }^{57} \mathrm{Co}$ and ${ }^{241} \mathrm{Am}$ sources show that the gain varies from 12.5 at $5.89 \mathrm{keV}$ to 11.3 at $59.54 \mathrm{keV}$ (see fig. 1). For visible light, a gain of $\sim 12.8$ has been measured with different wavelength LEDs. The only paper which reports similar results we know of is the one by Pansart [6]. Since the ratio of ionization by holes and electrons in the avalanche region is small $(\sim 0.01)$, only the X-rays absorbed before the high field region will generate pulses which will be amplified (see fig. 2). If the photoelectron is partially absorbed in the avalanche region, the gain is substantially lower. However this effect doesn't explain the photon energy dependence of the



Fig. 1 Gain of the APD 55345 as a function of the photon energy (bias voltage of $-260 \mathrm{~V}$ ).

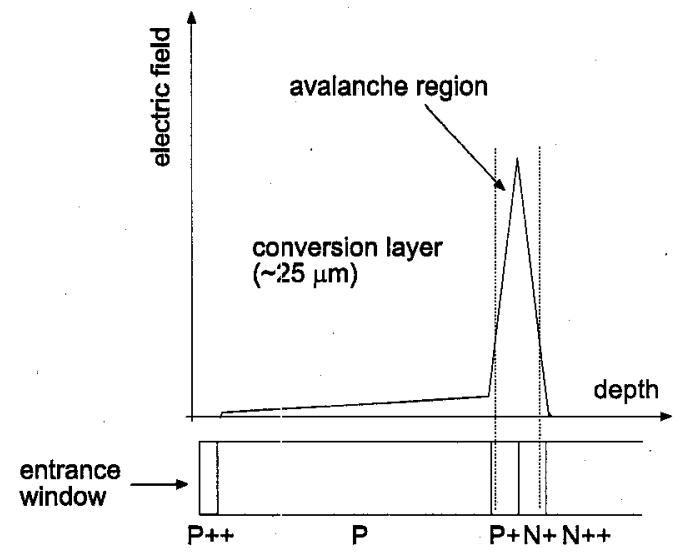

Fig. 2 Electric field profile of the APD S5345 (not to scale).

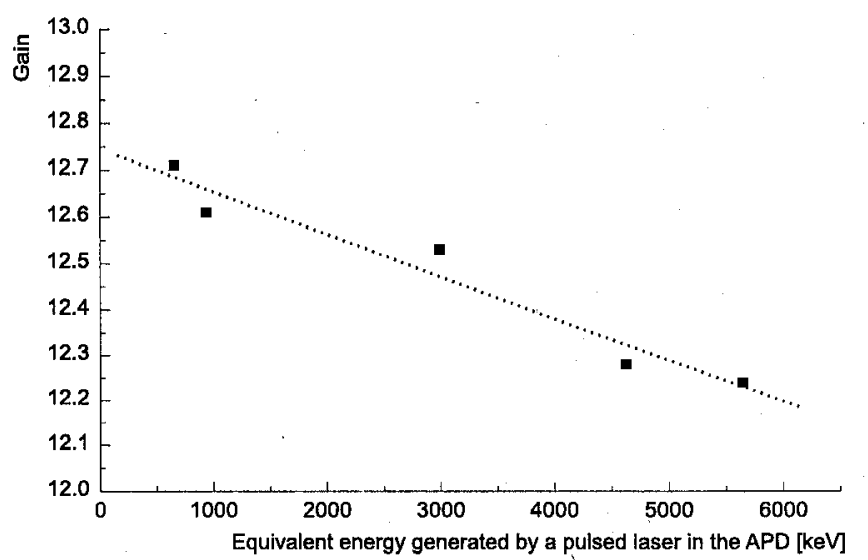

Fig. 3 Gain of the APD S5345 as a function of the equivalent energy generated by a $675 \mathrm{~nm}$ pulsed laser onto a $\sim 0.2 \mathrm{~mm}$ diameter area (bias voltage of $-260 \mathrm{~V}$ ).

gain since there is a difference between the gains of $5.89 \mathrm{keV}$ $\mathrm{X}$-rays and visible light photons which both deposit the total energy very close to the interaction point. An other explanation is that the charge clouds produced by X-ray interactions may disturb the high field in the avalanche region and thus decrease the gain. A comparable gain effect is present when the APD is illuminated with visible light. We used a $675 \mathrm{~nm}$ pulsed laser and find that the gain can vary from 12.7 to 12.2 depending on the light intensity (see fig. 3). With the laser, the light is focused onto a constant $\sim 50,000 \mu \mathrm{m}^{2}$ area. Then the gain seems to present a linear dependence with the charge density or with the total charge. With X-rays, the interaction is more or less 'pointlike' and no simple relation between the gain and the charge density or the total charge can be found. 
Table 2

FWHM measured on the pulse height spectrum of ${ }^{55} \mathrm{Fe}$ and ${ }^{241} \mathrm{Am}$ at room temperature.

${ }^{*}$ ) X-rays collimated through a $1 \mathrm{~mm}$ diameter and $2 \mathrm{~mm}$ thick aluminium pinhole.

\begin{tabular}{|l||l|l|l|l|l|}
\hline detector & $\begin{array}{l}\text { shaping time } \\
(\mu \mathrm{s})\end{array}$ & $\begin{array}{l}\text { FWHM (keV) } \\
5.89 \mathrm{keV}\end{array}$ & $13.9 \mathrm{keV}$ & $17.8 \mathrm{keV}$ & $59.54 \mathrm{keV}$ \\
\hline \hline PIN photodiode & 3 & $/$ & 3 & 2.9 & 2.9 \\
\hline SDD & 0.5 & 1.2 & 1.2 & 1.3 & 1.2 \\
\hline APD S5345 & 0.5 & 1 & 1.6 & 2.1 & 5.4 \\
& & $0.9^{*}$ & $1.1^{*}$ & $1.9^{*}$ & $4.6^{*}$ \\
\hline
\end{tabular}

\section{X-RAY AND GAMMA-RAY DETECTION}

\section{A. Room Temperature Measurements}

At room temperature, the SDD can detect photons up to 60 $\mathrm{keV}$ with an energy resolution of $1.2 \mathrm{keV} \mathrm{FWHM}$ (see fig. 4 and table 2). The performance of this detector is determined by the total capacitance. With a commercial 2SK147 JFET instead of the bare DUT JFET (specially designed to match the detector capacitance) we found an energy resolution of $2.4 \mathrm{keV}$ FWHM. Lechner reported an energy resolution of $225 \mathrm{eV} \mathrm{FWHM}$ at the $5.89 \mathrm{keV} \mathrm{K} \alpha$ peak of ${ }^{55} \mathrm{Fe}$ decay [4] obtained with a smaller SDD of only $3.5 \mathrm{~mm}^{2}$ active area with an integrated n-JFET.

With the APD, we measured at the $13.9 \mathrm{keV}$ peak of ${ }^{241} \mathrm{Am}$ a FWHM of $1.6 \mathrm{keV}$. Better results have been obtained with the APD at lower energies. A FWHM of $1 \mathrm{keV}$ has been measured at the $5.89 \mathrm{keV} \mathrm{K} \alpha$ peak of ${ }^{55} \mathrm{Fe}$ decay, and $1.1 \mathrm{keV}$ at the $6.4 \mathrm{keV} \mathrm{K} \alpha$ peak of ${ }^{57} \mathrm{Co}$ decay. The energy resolution of the APD is degraded by the non-uniformity of the response over the detection area. This has been checked by collimating the $\mathrm{X}$-rays through a $1 \mathrm{~mm}$ diameter and $2 \mathrm{~mm}$ thick aluminium pinhole. We then measured better FWHMs (see table 2) which are in agreement with equation (3) taking into account figure 1. The non-uniformity of the response has been confirmed by scanning the active area with a pulsed laser. Deviations of $10 \%$ can be observed (see fig. 5). Above $14 \mathrm{keV}$ the increase of the noise as a function of the energy is higher than what is expected from equation (3) taking into account figure 1 and the non-uniformity of the response. The fluctuation of the gain could be higher than what is described by the excess noise factor if charge cloud effects disturb the field in the avalanche region.

At the $59.54 \mathrm{keV}$ peak of ${ }^{241} \mathrm{Am}$ we measured with the PIN photodiode a FWHM of $2.9 \mathrm{keV}$ which is better than what is obtained with the APD. Obviously the PIN photodiode and the SDD don't suffer from a degradation of the FWHM with the photon energy caused by the variation of the gain.

The PIN photodiode and the SDD are fully depleted, the depletion layer being about $500 \mu \mathrm{m}$ thick, while in the APD pulses are generated when X-ray interaction occurs in the first 25 micrometers (see fig. 2). There is a difference in efficiency between the APD and the two other detectors which can be calculated from the photoelectric absorption probability. This ratio doesn't exceed a factor of 5 for photon energies under $10 \mathrm{keV}$ and reaches a maximum of a factor of 20 (the active thickness ratio) for photon energy above $20 \mathrm{keV}$.

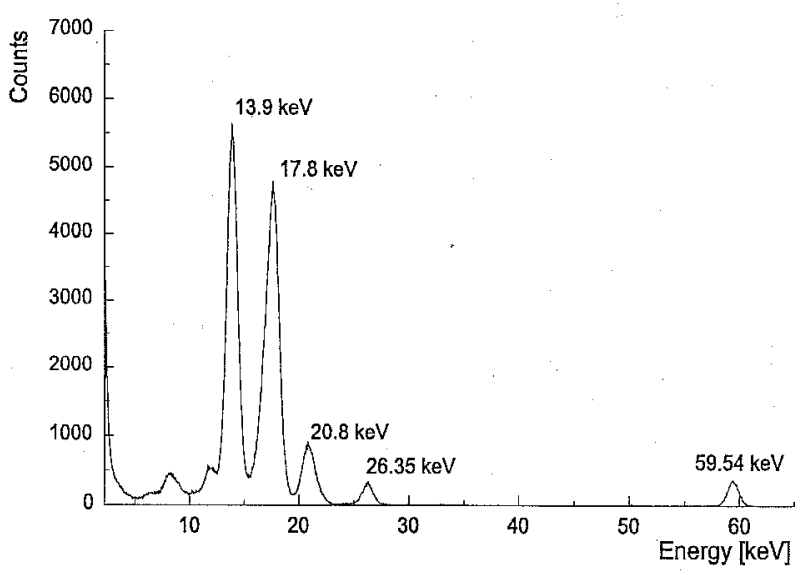

Fig. 4 Pulse height spectrum of ${ }^{241}$ Am measured with the SDD at room temperature.

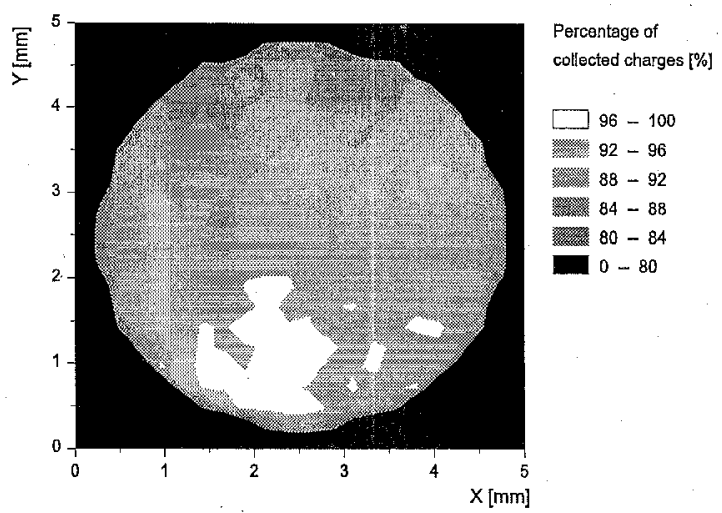

Fig. 5 Optical response to a $675 \mathrm{~nm}$ pulsed laser over the active area of the Hamamatsu S5345 APD.

\section{B. Cooled Detectors}

The measurements were carried out in a low temperature setup in which the temperature can be reduced from room temperature to $-70{ }^{\circ} \mathrm{C}$ and can be controlled within $\pm 0.2{ }^{\circ} \mathrm{C}$. By cooling the detector, the leakage current is reduced roughly by a factor 2 every $10^{\circ} \mathrm{C}$ decrease in temperature. With the PIN photodiode and the SDD, it is thus possible to decrease the temperature enough so that the leakage current is no more a main contribution to the noise. These detectors are then limited by the junction capacitance and the dielectric losses [5]. This explains the large difference between the results obtained with the PIN photodiode and the SDD (see tables 1 and 3). At low temperature, the best results are obtained 
Table 3

FWHM measured at the $5.89 \mathrm{keV} \mathrm{K \alpha}$ peak of ${ }^{55} \mathrm{Fe}$ decay at low temperature.

$\left(^{*}\right) \mathrm{X}$-rays collimated through a $2 \mathrm{~mm}$ diameter and $2 \mathrm{~mm}$ thick aluminium pinhole.

\begin{tabular}{|l||l|l|l|l|}
\hline detector & $\begin{array}{l}\text { temperature } \\
\left({ }^{\circ} \mathrm{C}\right)\end{array}$ & bias voltage $(\mathrm{V})$ & $\begin{array}{l}\text { shaping } \\
\text { time }(\mu \mathrm{s})\end{array}$ & FWHM (keV) \\
\hline \hline PIN photodiode & -72 & -50 & $3-10$ & 1.7 \\
\hline SDD & -65 & -230 & 6 & 0.19 \\
\hline APD S5345 & -13 & -260 & 0.5 & 0.81 \\
& & & $0.54^{*}$ \\
\hline
\end{tabular}

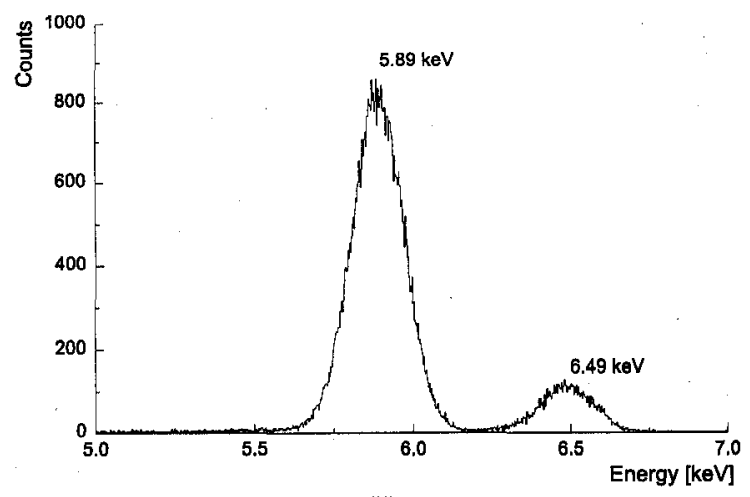

Fig. 6 Pulse height spectrum of ${ }^{55} \mathrm{Fe}$ measured at $-65^{\circ} \mathrm{C}$ with the SDD. The FWHM at the $5.89 \mathrm{keV} \mathrm{K} \alpha$ peak of ${ }^{55} \mathrm{Fe}$ decay is $193 \mathrm{eV}$. The number of noise electrons $N_{e}$ is 18.5 .

with the circular SDD. The small junction capacitance of this detector allows to obtain a FWHM of $193 \mathrm{eV}$ at $-65{ }^{\circ} \mathrm{C}$ at the $5.89 \mathrm{keV} \mathrm{K} \alpha$ peak of ${ }^{55} \mathrm{Fe}$ decay (see fig. 6). It must be noticed that this detector, slightly cooled to $-15^{\circ} \mathrm{C}$ has a FWHM of $290 \mathrm{eV}$. Gauthier measured an energy resolution of $143 \mathrm{eV}$ FWHM with a SDD whith an active area of about $1 \mathrm{~cm}^{2}$ but with a longer shaping time of $30 \mu \mathrm{s}$ and at a lower temperature of $-120^{\circ} \mathrm{C}$ [3]. The APD should not be cooled under $-20{ }^{\circ} \mathrm{C}$ according to Hamamatsu specifications [15], but the optimum temperature was about $-15^{\circ} \mathrm{C}$. The gain of the APD is temperature dependent due to the temperature dependence of the electron mobility. We found a value for the variation of about $-2 \% /{ }^{\circ} \mathrm{C}$. At $-13{ }^{\circ} \mathrm{C}$, the gain is 1.6 times higher than at room temperature and we measured a FWHM of $810 \mathrm{eV}$ at the $5.89 \mathrm{keV} \mathrm{K} \alpha$ peak of ${ }^{55} \mathrm{Fe}$ decay. The energy resolution can be improved by using a $2 \mathrm{~mm}$ diameter and 2 $\mathrm{mm}$ thick aluminium pinhole. We then measured a FWHM of $540 \mathrm{eV}$.

\section{REAdOUT OF CSI(TL) SCINTILLATION LIGHT}

In all scintillator measurements we used a CsI(Tl) scintillator with a diameter of $5 \mathrm{~mm}$ and a height of $7.5 \mathrm{~mm}$, coated with four layers of teflon tape. The light yield of this CsI(Tl) crystal is estimated to be $50,000 \mathrm{ph} / \mathrm{MeV}$ at $10 \mu \mathrm{s}$ shaping time and $45,000 \mathrm{ph} / \mathrm{MeV}$ at $6 \mu$ s shaping time. The emission is maximum at $565 \mathrm{~nm} .{ }^{137} \mathrm{Cs} \gamma$-rays have been measured with a combination of the CsI(Tl) scintillator and the different detectors. All the results and parameters are summarized in table 4.
For the PIN photodiode and the SDD, the photon detection efficiency was calculated from the position of the $662 \mathrm{keV}$ peak of ${ }^{137} \mathrm{Cs}$ compared to the position of the $59.54 \mathrm{keV}$ peak of ${ }^{241} \mathrm{Am}$, directly detected in silicon. For the Hamamatsu S5345 APD (see fig. 7) the gain is energy dependent. Consenquently direct X-ray interaction can not be used as a reference to calculate accurately the number of electron-holes pairs created from scintillation light. We calibrated the electronic chain with pulses fed through the test capacitor of the preamplifier. The photon detection efficiency can then be calculated from the photopeak position knowing that the gain for visible light is about 12.8 at a bias voltage of $-260 \mathrm{~V}$. Unfortunately, the crystal we used presents a scintillator resolution higher than $5 \%$ (see equation (4) and table 4). We measured therefore energy resolutions which are slightly worse than what can be found in literature $[1,22,23]$. Fiorini reported at the conference of Elba (May 1997) a FWHM of $4.34 \%$ at the $662 \mathrm{keV}$ peak of ${ }^{137} \mathrm{Cs}$ obtained with a SDD. Although our resolution is not as good as this, our measurements, since they are made with the same crystal, allow a comparison between the silicon detectors and PMMTs (see table 4). Both the SDD and the APD have a slightly better energy resolution than what is obtained with a normal PMT XP2020Q, and equal to what is obtained with the red sensitive PMT XP2254B. With the PIN photodiode we measured energy resolution slightly worse than what is obtained with the P'MT XP2020Q. The silicon detectors present a higher photon detection efficiency than PMTs in the emission range of $\mathrm{CsI}(\mathrm{Tl})(450-650 \mathrm{~nm})$. For silicon detectors

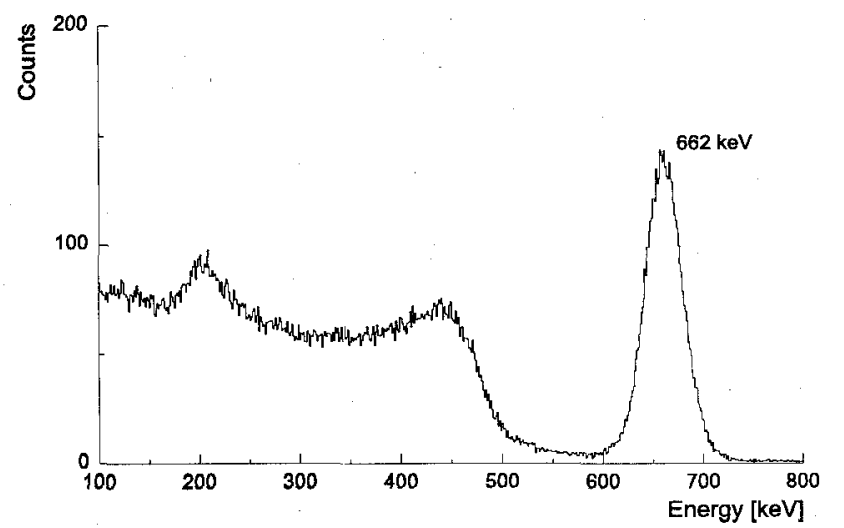

Fig. 7 Pulse height spectrum of ${ }^{137} \mathrm{Cs}$ measured with a combination of CsI(Tl) and the Hamamatsu \$5345 APD. 
Table 4

Measurements of CsI(Tl) scintillation light. $R_{d}$ and $R_{\varepsilon}$ are respectively the detector and scintillator resolution.

\begin{tabular}{|l||l|l|l|l|l|l|}
\hline detector & $\begin{array}{l}\text { shaping } \\
\text { time }(\mu \mathrm{s})\end{array}$ & $\begin{array}{l}\text { energy resolution at } \\
\text { the } 662 \mathrm{keV} \text { peak of } \\
\mathbf{1 3 7} \mathrm{Cs}(\%)\end{array}$ & $\begin{array}{l}R_{d} \\
(\%)\end{array}$ & $\begin{array}{l}R_{s} \\
(\%)\end{array}$ & $\begin{array}{l}\text { photon detection } \\
\text { efficiency }(\%)\end{array}$ & $\begin{array}{l}\mathrm{e}-\mathrm{h} \text { pairs/MeV or } \\
\text { phe/MeV }\end{array}$ \\
\hline \hline PIN photodiode & 6 & 7.9 & 5.2 & 5.6 & 55 & 25000 \\
\hline SDD & 6 & 6.6 & $/$ & $/$ & 55 & 24700 \\
\hline APD S5345 & 6 & 6.6 & 3.3 & 5.7 & 69 & 31300 \\
\hline PMT XP2020Q & 10 & 7.1 & 4.9 & 5.1 & 8 & 3900 \\
\hline PMT XP2254B & 10 & 6.6 & 3.2 & 5.8 & 18 & 9100 \\
\hline
\end{tabular}

the photon detection efficiency varies from $55 \%$ to $69 \%$ while for the normal PMT and the red sensitive PMT we measured respectively about $8 \%$ and $18 \%$.

\section{CONCLUSION}

For the direct detection of X-rays as well as for the detection of $\mathrm{CsI}(\mathrm{Tl})$ scintillation light, the best energy resolution is obtained with the SDD. High efficiency, large detection area, position-sensitivity and good energy resolution make this detector suitable for many applications. The disadvantage of this detector is the charge collection time which increases with active area. For time resolved detection, one should prefer the APD S5345 which presents good features for soft X-rays and scintillation light detection. The PIN photodiode is a low budget alternative, good enough for many applications.

\section{ACKNOWLEDGMENTS}

The authors would like to thank J. Ligthart, G.W. Lubking, P.M. Sarro and J.C. van 't Spijker. These investigations have been supported by the Netherlands Technology Foundation (STW).

\section{REFERENCES}

[1] R. Farrell, F. Olschner et al., "Advances in semiconductor photodetectors for scintillators", Nucl. Instr. and Meth., A 387, pp. 194-198, 1997.

[2] E. Gatti, P. Rehak, "Semiconductor drift chamber on application of novel charge transport scheme", Nucl. Instri and Meth., A 225, pp. 608-614, 1984

[3] Ch. Gauthier, J. Goulon et al., "A high-resolution silicon drift chamber for X-ray spectroscopy", Nucl. Instr. and Meth., A 349 , pp. 258-262, 1994.

[4] P. Lechner, S, Eckbauer et al., "Silicon drift detectors for high resolution room temperature X-ray spectroscopy", $\mathrm{Nucl}$. Instr. and Meth., A 377, pp. 346-351, 1996.

[5] A. Pullia, G. Bertuccio, "Resolution limits of silicon detectors and electronics for soft X-ray spectroscopy at non cryogenic temperatures", Nucl. Instr. and Meth., A 380, pp. 1-5, 1996.

[6] J.P. Pansart, "Avalanche photodiodes for particle detection", Nucl. Instr. and Meth., A 387, pp. 186-193, 1997.

[7] R.J McIntyre, "Multiplication Noise in Uniform Avalanches Photodiodes", IEEE Trans. Electron. Dev., ED-13, pp. 164-168, 1966.
[8] P.P. Webb, R.J McIntyre, "Properties of avalanche photodiode.", RCA Review, vol. 35, pp. 234-278, 1974.

[9] A. Ochi, Y. Nishi, T. Tanimori, "Study of a large avalanche photodiode as a fast photon and soft X-ray detector", Nucl. Instr. and Meth., A 378, pp. 267-274, 1996.

[10] A.Q.R. Baron, S.L. Ruby, "Time resolved detection of X-rays using large area avalanche photodiode", Nucl. Instr. and Meth., A 343, pp. 517-526, 1994.

[11] R. Lecomte, C. Martel, C. Carrier, "Status of BGOavalanche photodiode detectors for spectroscopic and timing measurements", Nucl. Instr. and Meth., A 278, pp. 585-597, 1989.

[12] C. Carriet, R. Lecomte, "Recent Results in Scintillation Detection with Silicon Avalanche Photodiodes", IEEE Trans. Nucl. Sci., NS-37, pp. 209-214, 1990.

[13] I. Holl, E. Lorenz et al., "Some Studies of Avalanche Photodiode Readout of Fast Scintillators", IEEE Trans. Nucl. Sci., NS-42, pp. 351-356, 1995.

[14] G. Bertuccio, A. Pullia, "Room temperature X-ray spectroscopy with a silicon diode Detector and an Ultra Low Noise Preamplifier", IEEE Trans, Nucl. Sci., NS-41, pp. 1704-1709, 1994.

[15] "Hamamatsu photodiodes", dec. 1995 T Cat. No. KPD $0001 E 04$. Hamamatsu Photonics K.K., Solid State Division 1126-1, Ichinocho, Hamamatsu City, 435-91, Japan.

[16] G. Bertuccio, L. Fasoli et al., "Spectroscopy Charge Amplifier for Detectors with Integrated Front-End FET", IEEE Trans. Nucl. Sci., NS-42, pp. 1399-1405, 1995.

[17] G. Bertolini, A. Coche, Semiconductor detectors, Amsterdam: Elsevier-Notth Holland, 1968.

[18] F.S. Goulding, D.A. Landis, Nuclear Spectroscopy and Reactions, vol. 40, pp. 413-468, 1974.

[19] E. Gatti, P.F. Manfredi et al.,."Suboptimal filtering of $1 / f$-noise in detector charge measurements", Nucl. Instr. and Meth., A 297, pp. 467-478, 1990.

[20] H.R. Zulliger, D.W. Aikten, "Fano factor fact and fallacy", IEEE Trans. Nucl. Sci. NS-17, pp. 187-195, 1970

[21] P. Dorenbos et al., "Non-Proportionality in the Scintillation Response and the Energy Resolution Obtainable with Scintillation Crystals", IEEE Trans. Nucl. Sci., NS-42, pp. 2190-2202, 1995

[22] E. Gramsh, K.G. Lynn, M. Weber, "Silicon PIN photodetectors in high resolution nuclear spectroscopy", Nucl. Instr. and Meth., A 311,pp. 529-538, 1992.

[23] E. Sakai, "Recent Measurements on Scintillation-Photodetector Systems", IEEE Trans. Nucl. Sci., NS-34, p. 418, 1987. 